\title{
Deriving Item Features Relevance from Past User Interactions
}

\author{
Leonardo Cella \\ leonardo.cella@mail.polimi.it \\ Politecnico di Milano \\ Massimo Quadrana \\ massimo.quadrana@polimi.it \\ Politecnico di Milano
}

\author{
Stefano Cereda \\ stefano1.cereda@mail.polimi.it \\ Politecnico di Milano \\ Paolo Cremonesi \\ paolo.cremonesi@polimi.it \\ Politecnico di Milano
}

\begin{abstract}
Item-based recommender systems suggest products based on the similarities between items computed either from past user preferences (collaborative filtering) or from item content features (contentbased filtering). Collaborative filtering has been proven to outperform content-based filtering in a variety of scenarios. However, in item cold-start, collaborative filtering cannot be used directly since past user interactions are not available for the newly added items. Hence, content-based filtering is usually the only viable option left.

In this paper we propose a novel feature-based machine learning model that addresses the item cold-start problem by jointly exploiting item content features and past user preferences. The model learns the relevance of each content feature from the collaborative item similarity, hence allowing to embed collaborative knowledge into a purely content-based algorithm. In our experiments, the proposed approach outperforms classical content-based filtering on an enriched version of the Netflix dataset, showing that collaborative knowledge can be effectively embedded into content-based approaches and exploited in item cold-start recommendation.
\end{abstract}

\section{INTRODUCTION}

Item-based algorithms are widespread methods for recommending relevant items to users [4]. Because predictions rely on the computation of similarities between pairs of items, they have good runtime performance and their recommendations are easy to explain. Collaborative Filtering (CF) similarities usually leads to good predictive accuracy, especially if trained with regard to suitable optimization objective functions [3]. The downside of CF algorithms is that similarities are only available for items with a - possibly large - number of ratings. Thus for entirely new items - i.e., ones that have no ratings - CF methods are not capable of computing recommendations. Moreover, $\mathrm{CF}$ are biased toward popular Blockbuster items, thus reducing the chances of novel recommendations. This happens because algorithms are trained (e.g., tuned) to achieve the best performance in terms of available ratings. This creates the rich-get-richer effect for popular items, which results in reducing the coverage of recommendations.

Permission to make digital or hard copies of all or part of this work for personal or classroom use is granted without fee provided that copies are not made or distributed for profit or commercial advantage and that copies bear this notice and the full citation on the first page. Copyrights for components of this work owned by others than ACM must be honored. Abstracting with credit is permitted. To copy otherwise, or republish, to post on servers or to redistribute to lists, requires prior specific permission and/or a fee. Request permissions from permissions@acm.org.

UMAP'17, fuly 09-12, 2017, Bratislava, Slovakia

(c) 2017 ACM. 978-1-4503-4635-1/17/07 . \$15.00

DOI: http://dx.doi.org/10.1145/3079628.3079695
The new-item problem can be solved by using Content-Based Filtering methods $(\mathrm{CBF})$. $\mathrm{CBF}$ requires that items are represented via a set of features - or attributes - that capture their intrinsic characteristics. The quality of $\mathrm{CBF}$ is severely limited by three factors [7]:

- quality is linked to the availability of a number of significant, well-structured, editorial-generated attributes (such as genres, actors, directors in the movie domain); however, many recommender systems base their recommendations on unstructured, user-generated attributes (i.e., tags, reviews) most of which are noisy or scarcely relevant;

- recommendations to each user do not use the ratings of other users, therefore ignoring potentially useful collaborative information;

- recommended items are similar to previously rated items, thus reducing diversity of recommendations.

Because of these limitations, a significant challenge to address with $\mathrm{CBF}$ is feature weighting, i.e., how to identify how much a feature is important in defining the perceived similarity between items.

Feature weighting can be viewed as an extension of the feature selection problem, where the goal is to provide an evaluation measure used to score and filter the different features. The choice of evaluation distinguishes between the three main categories of feature weighting algorithms: filters, embedded methods and wrappers [2].

Filter methods produce a feature set which is not tuned to a specific type of rating predictive model. Filter methods use a proxy measure instead of the error rate to score a feature subset. Common filters in recommender systems are based on TF-IDF weighting schemes [6].

Embedded methods perform feature weighting as part of the rating model construction process. Examples in recommender systems are Factorization Machines [10], UFSM [1] and SSLIM [8].

Wrapper methods use a pre-trained CF predictive model to score features, with a two-step approach. A CF algorithm is used to produce a model (step 1) and the wrapper methodology consists in using the CF model to assess the relative usefulness of features (step 2).

The main contributions of this work is a general, simple and straightforward wrapper method to make content-based algorithms ratings-aware by plugging learnable attribute weights onto them. The core principle is that if a feature co-occurs frequently only within similarly co-rated items, then that feature is a good candidate to be included as a relevant feature in defining the similarity between items. 
We conducted two experimental evaluations of the proposed method using the Netflix dataset enriched with attributes from IMDB. The experiment results show that the proposed model outperforms state-of-art CBF in both the warm-start and new-item scenarios.

\section{RELATED WORKS}

Some attempts have been done to reduce the quality gap between $\mathrm{CF}$ and $\mathrm{CBF}$. Past works operate along two directions: filtering and embedded methods.

Filtering methods have underpinnings in information theory and information retrieval [6] and evaluate features without involving any learning algorithm. Filtering methods weight features with the goal of maximizing the information gain (e.g., TF-IDF or entropy-based weighting) and mitigating synonymy (e.g., LSA). As an example, the work in [9] adopts a TF-IDF approach in which users are considered as documents and the TF-IDF component is normalized across all users. The main drawback of filtering methods is that they do not take into account the ratings of users, therefore ignoring if the feature-based similarity between items is aligned with the user perception of similarity.

Embedded methods incorporate feature weighting as part of the rating learning process, and use the objective function of the learning process to guide searching for relevant features. Examples in recommender systems are SSLIM [8], UFSM [1] and Factorization Machines [10]. SSLIM adopts a Sparse Linear method with Side information for learning a sparse similarity matrix that is used for computing top-N recommendations [8]. The training incorporate both ratings from users and features about items. The main drawback of this approach is that the similarity is computed directly, without computing feature weights, and therefore cannot be used for new items. User-Specific Feature-based Similarity Models (UFSM) learn a personalized linear combination of similarity functions known as global similarity functions for cold-start top-N item recommendations. UFSM can be considered as a special case of Factorization Machines [1]. The main drawback of embedded methods is the coupling between the collaborative and content components of the model. When used on datasets with unstructured user-generated features (e.g., tags) the noise from the features propagate to the collaborative part, affecting the overall quality of the model. For this reason, when used in the new item scenario, predictive accuracy is only marginally improved with respect to content based techniques.

\section{LEAST SQUARES FEATURE WEIGHING}

As in any recommender system, we consider the problem of recommending items from a set $I$ to users in the set $U$, and item features are picked from a set $F . \mathbf{R}^{|U| x|I|}$ is the feedback matrix (either explicit or implicit). $\mathrm{A}^{|I| x|F|}$ is the binary item feature matrix, in which $a_{i f}=1$ iff item $i$ has feature $f$. In a generic item similarity model, given a item similarity matrix $S^{|I| x|I|}$, the predicted relevance $\hat{r}_{u i}$ of item $i$ for user $u$ is computed as

$$
\hat{r}_{u i}=\sum_{j \in N_{k}(i)} r_{u j} s_{i j}
$$

where $N_{k}(i)$ is the set of $k$ nearest neighbors of item $i$ according to the similarity model. In top- $N$ recommendation, the $N$ items with the largest predicted relevance are recommended to the user. Notice that this approach allows to estimate the predicted rating for any item $i$ as long as the similarities between $i$ and any other item $j \in I$ can be computed, new items included.

Feature weighing. In its simplest formulation, feature weighing consists in computing the array of weights $\mathbf{w} \in \mathbb{R}^{|F|}$ such that each entry $w_{f} \in \mathbf{w}$ reflects the importance of the feature $f \in F$ for the given task. In other words, if two features $f$ and $f^{\prime}$ have weights $w_{f}>w_{f^{\prime}}$, then feature $f$ is more relevant than $f^{\prime}$ for the task. We define the weighted similarity $s_{i j}$ between items $i$ and $j$ as

$$
s_{i j}^{(w)}=\sum_{f \in F} w_{f} a_{i f} a_{j f}=\left\langle\mathbf{w}, \mathbf{a}_{i} \odot \mathbf{a}_{j}\right\rangle
$$

where $\mathbf{a}_{i}, \mathbf{a}_{j} \in\{0,1\}^{|F|}$ are the feature vectors of items $i$ and $j$ respectively and $\odot$ is the element-wise product.

In the case of binary attribute matrices, a typical feature weighting scheme is TF-IDF that weights the features in $F$ proportionally to their specificity.

The proposed approach. In this work, we propose a solution based on least squares (LSQ) optimization to automatic feature weighing. Automatic feature weighing aims at inferring the weights in $\mathbf{w}$ from a collaborative model. Given the item-to-item weight matrix $S^{(\mathrm{CF})} \in \mathbb{R}^{|I| x|I|}$ computed with collaborative filtering, the optimal weight vector $\mathbf{w}^{*}$ can be determined by solving the following LSQ problem:

$$
\underset{\mathbf{w} *}{\operatorname{argmin}}\left\|S^{(\mathrm{CF})}-S^{(\mathrm{w})}\right\|_{F}^{2}
$$

where $S^{(\mathrm{w})} \in \mathbb{R}^{|I| x|I|}$ is the pairwise weighted similarity matrix computed with (2). This LSQ formulation allows to compute the optimal weight vector $\mathrm{w}^{*}$ capable to reconstruct the $\mathrm{CF}$ similarity matrix by means of simple weighted CBF similarity with minimal error. Since our goal is to learn a set of feature weights so that $\mathrm{CBF}$ similarities mimic as close as possible $\mathrm{CF}$ ones, there is no need to add a regularization term, thus greatly simplifying the optimization. In the case of the simple linear weighing scheme of (2), the problem boils down to the following simple linear regression

$$
\underset{\mathbf{w} *}{\operatorname{argmin}} \sum_{i \in I} \sum_{j \in I \backslash\{i\}}\left(s_{i j}^{(C F)}-\left\langle\mathbf{w}, \mathbf{a}_{i} \odot \mathbf{a}_{j}\right\rangle\right)^{2}
$$

which can be efficiently solved analytically. We call our approach LFW (Least-squares Feature Weighing).

When a new item is added to the catalog, we use $\mathbf{w}^{*}$ to compute its weighted similarity w.r.t. the previously existing items. Then, it can be recommended to users by using Equation 1. We conjecture that our model is capable to weight features in accordance to their collaborative relevance. If this conjecture holds, the learned weighting scheme should outperform traditional, fully contentbased weighting schemes (like TF-IDF) in the new item scenario without exhibiting a severe degradation of performance in standard (warm-start) scenario.

It is worth noting that the collaborative similarity matrix $S^{(\mathrm{CF})}$ can be obtained with any $\mathrm{CF}$ algorithm. In our experiments, we 
used SLIM since it has been extensively proven to achieve state-ofthe-art performance in many CF tasks [11].

\section{EVALUATION}

We performed experiments to confirm that our approach (a) is capable to produce useful recommendations in an item cold-start scenario without (b) incurring into severe degradation of performance in warm-start scenarios.

Dataset. For our experiments, we used a version of the Netflix dataset enriched with structured and unstructured attributes extracted from IMDB. This dataset has $250 \mathrm{k}$ users, $6.5 \mathrm{k}$ movies and $8.8 \mathrm{M}$ ratings in 1-5 scale. The rating data is enriched with 4699 binary attributes representing various kinds of meta-information on movies such as director, actor, genres and user-generated tags.

Evaluation procedure. To investigate the new-item scenario, we performed a 70/30 random hold-out split over items. To investigate the item warm-start scenario, we instead split over users with the same proportions. The final partitioning of the dataset is show in Figure 1. The sub-matrix A was used to train SLIM first, and then to fit SLIM similarities with our LFW model. The hyper-parameters of SLIM were tuned on a separate validation set extracted from A before fitting LFW. The neighborhood size $k$ was tuned on a second validations set extracted from B by using ROC-AUC. The models fit on $\mathrm{A}$ were used to generate recommendations both in the new-item and in the warm-start scenario.

Sub-matrices B and C were used exclusively in evaluation. In the new-item scenario, the ratings in A were used as user-profile to score the items in C, the ground truth. In the warm-start scenario, we held-out $30 \%$ of the positive ratings $(>3)$ of every user as ground truth and used the remaining ratings as user profiles. In both scenarios, the ground truth is composed only by items with rating $>3$. We used accuracy metrics (Precision, Recall, MRR, MAP and NDCG) to evaluate the ranking recommendation quality. We also evaluate the Coverage and Diversity in recommendation by using the definitions in [5]. Notice that, since the user profiles and ground truth differ in the two scenarios analyzed, their results are not directly comparable.

Baselines. We used simple unweighted cosine similarity (Cosine) and TF-IDF-weighted cosine similarity (CosineIDF) as CBF baselines to evaluate the performance of LFW in both scenarios ${ }^{1}$. SLIM was used as additional CF baseline in the warm-start scenario.

Figure 1: Dataset partitioning with dimensions.

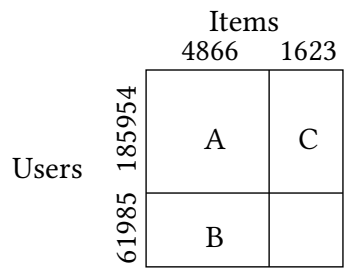

\footnotetext{
${ }^{1}$ We experimented also with other similarity metrics (e.g. Pearson), but those were consistently outperformed by these baselines, so we do not report them here.
}

Discussion. Let us discuss the new-item scenario first, since it is the main focus of our work. In Table 1, we report the accuracy metrics for different recommendation list sizes $N$. LFW consistently outperforms all the baselines in all metrics at any value of $N$. We want to highlight that LFW differs from the other CBF baselines solely in the feature weighing scheme. Therefore, the improvement in performance must be due to a better feature weighing discovered by our approach.

In Table 2, we report the results for the item warm-start scenario. For space reasons, we report only the values for $N=5$. As expected, the CF approach based on SLIM has the best predictive accuracy in the warm-start scenario. Interestingly, LFW still outperforms both Cosine and Cosine TF-IDF baselines by a even large margin with respect to the new item scenario. In summary, LFW cannot compete with SLIM in warm-start scenarios, also due to its CBF nature. Still, its degradation in performance is much less evident than the other CBF baselines.

Table 1: Performance evaluation for the new-item scenario.

\begin{tabular}{ccccccc}
\hline Algorithm & $n$ & Precision & Recall & MRR & MAP & NCDG \\
\hline \multirow{4}{*}{ LFW } & 5 & $\mathbf{0 . 1 3 2 3}$ & $\mathbf{0 . 0 8 2 5}$ & $\mathbf{0 . 2 5 5 1}$ & $\mathbf{0 . 1 0 6 2}$ & $\mathbf{0 . 0 9 4 0}$ \\
& 25 & $\mathbf{0 . 0 8 5 8}$ & $\mathbf{0 . 2 4 0 6}$ & $\mathbf{0 . 2 7 9 5}$ & $\mathbf{0 . 0 9 1 5}$ & $\mathbf{0 . 1 7 1 5}$ \\
& 50 & $\mathbf{0 . 0 6 5 4}$ & $\mathbf{0 . 3 4 5 9}$ & $\mathbf{0 . 2 8 2 2}$ & $\mathbf{0 . 0 9 5 0}$ & $\mathbf{0 . 2 0 9 7}$ \\
& & & & & & \\
Cosine & 5 & 0.0938 & 0.0502 & 0.1984 & 0.0748 & 0.0622 \\
& 25 & 0.0624 & 0.1473 & 0.2189 & 0.0573 & 0.1127 \\
& 50 & 0.0485 & 0.2231 & 0.2218 & 0.0582 & 0.1411 \\
CosineIDF & 25 & 0.0658 & 0.1564 & 0.2280 & 0.0605 & 0.1192 \\
& 50 & 0.0510 & 0.2348 & 0.2309 & 0.0621 & 0.1486 \\
\hline
\end{tabular}

Table 2: Performance evaluation for the warm-start scenario.

\begin{tabular}{ccccccc}
\hline Algorithm & $N$ & Precision & Recall & MRR & MAP & NCDG \\
\hline SLIM & 5 & $\mathbf{0 . 1 3 8 3}$ & $\mathbf{0 . 1 4 9 7}$ & $\mathbf{0 . 2 8 1 9}$ & $\mathbf{0 . 1 3 4 2}$ & $\mathbf{0 . 1 4 5 3}$ \\
LFW & 5 & $\underline{0.0640}$ & $\underline{0.0577}$ & $\underline{0.1438}$ & $\underline{0.0744}$ & $\underline{0.0597}$ \\
Cosine & 5 & 0.0334 & 0.0277 & 0.0826 & 0.0270 & 0.0302 \\
CosineIDF & 5 & 0.0337 & 0.0258 & 0.0855 & 0.0276 & 0.0296 \\
\hline
\end{tabular}

Table 3: Evaluation of coverage and diversity.

\begin{tabular}{ccc|cc}
\hline \multirow{2}{*}{ Algorithm } & \multicolumn{2}{c}{ new-item } & \multicolumn{2}{c}{ warm-start } \\
& Coverage & Diversity & Coverage & Diversity \\
\hline SLIM & - & - & 0.1683 & $\mathbf{4 . 0 5 8 5}$ \\
LFW & $\underline{0.8897}$ & $\underline{\mathbf{5 . 3 6 2 8}}$ & $\underline{0.4891}$ & $\underline{2.1289}$ \\
Cosine & 0.9236 & 4.1529 & $\mathbf{0 . 5 7 2 3}$ & 1.1519 \\
CosineIDF & $\mathbf{0 . 9 4 9 5}$ & 4.2061 & 0.5684 & 1.1517 \\
\hline
\end{tabular}


In Table 3, we report the evaluation of coverage and diversity for the top-5 recommendations in both scenarios. In the warm-start scenario, "traditional" $\mathrm{CBF}$ algorithms have greater coverage than $\mathrm{CF}$, whereas CF has greater diversity in the warm-start scenario. nterestingly, LFW seems to "take the best" from both worlds, achieving CBF-level coverage and CF-level diversity in both scenarios.

\section{QUALITATIVE ANALYSIS}

The good performances in both scenarios suggest that the weight vector $w^{*}$ is capable of highlighting the importance of the various features in terms of the user-perceived similarity. In order to understand this concept better, we provide a qualitative analysis on how features are weighted differently by two methods, namely Cosine TF-IDF and our method LFW.

In Table 4, we report the highest and lowest weighted features by LFW. For example, Jay Roach is the director of both "Meet the Parents" and "Meet the Fockers", and of the Austin Powers series. All these movies are comedy and target roughly the same audience, hence they are good candidates of being recommended by a $\mathrm{CF}$ algorithm despite having very different casts. Lois Maxwell has acted as Miss Moneypenny - James Bond's secretary - in the first 14 Bond movies. Hence, she represents another important cluster of movies. Interestingly, Lois Maxwell has higher importance than the various directors or main characters of Bond movies, since they changed frequently among them. On the other hand, the feature "snakebite" hardly identifies similar movies (for example, it is shared by completely different movies like "Kill Bill: Vol. 2" and "Siddharta"). Analogously, the feature "giant spider" is shared by two masterpieces ("The Lord of the Rings" and "Harry Potter") and a collection of 12 minor unrelated movies.

Table 4: Features relevance learned by LFW.

\begin{tabular}{cc}
\hline Most relevant features & Least relevant features \\
\hline Molly Ringwald & snakebite \\
Jay Roach & John Ashton \\
Lois Maxwell & Ally Sheedy \\
motorcycle accident & giant spider \\
Jayne Eastwood & stable \\
\hline
\end{tabular}

Table 5 shows that TF-IDF, by weighing features proportionally to their rarity, can hardly capture any interesting relationship from the actual movie consumption. Surprisingly, features representing genres (such as comedy and thriller) have low weights.

To shed a light on this, in Table 6 we report the features that differ the most between LFW and TF-IDF. The weights vectors of both methods were first normalized in $[0,1]$ before computing their difference feature-wise. It is interesting to see that LFW raises the relevance of genres w.r.t. TF-IDF, which are definitely an important set of features that receive a really low TF-IDF weight. Conversely, the relevance of very rare countries of origin in our dataset (e.g. Brazil and Romania) is strongly decreased. For example, we have only 2 Brazilian movies with just 20 ratings in common in our dataset.
Table 5: Features relevance according to TF-IDF.

\begin{tabular}{cc}
\hline Most relevant features & Least relevant features \\
\hline knowledge & drama \\
Iran & comedy \\
Bulgaria & film independent \\
China & thriller \\
Argentina & romance \\
\hline
\end{tabular}

Table 6: Feature importance LFW vs TF-IDF.

\begin{tabular}{cc}
\hline LFW $>$ TF-IDF & LFW $<$ TF-IDF \\
\hline USA & Brazil \\
drama & Romania \\
Molly Ringwald & Peru \\
comedy & Philippines \\
film independent & George P. Cosmatos \\
\hline
\end{tabular}

\section{CONCLUSION AND FUTURE WORK}

In this paper, we propose a feature-based linear regression framework for personalized recommendation. We presented a novel approach to compute item feature scores that defines their relevance according to expressed user preferences. In contrast to traditional recommender systems, our approach solves both the item and the user cold start issues. Moreover, it has a linear temporal complexity with respect to item features, and thus it is lighter than the state of the art competitor models. Future directions include the development of personalized feature weighing tools and the extensive evaluation of this approaches with different datasets and CF algorithms.

\section{REFERENCES}

[1] Asmaa Elbadrawy and George Karypis. 2015. User-Specific Feature-Based Similarity Models for Top-n Recommendation of New Items. ACM Trans. Intell. Syst. Technol. 6, 3, Article 33 (April 2015), 20 pages. DOI : http://dx.doi.org/10.1145/2700495

[2] Isabelle Guyon and André Elisseeff. 2003. An Introduction to Variable and Feature Selection. F. Mach. Learn. Res. 3 (March 2003), 1157-1182. http://dl.acm.org/ citation.cfm? id $=944919.944968$

[3] Domonkos Tikk Istvn Pilszy. 2009. Recommending new movies: even a few ratings are more valuable than metadata. RecSys 09 Proceedings of the third ACM conference on Recommender systems (2009), 93 - 100. DOI : http://dx.doi.org/10. $1145 / 1639714.1639731$

[4] Jon Herlocker Shilad Sen J. Ben Schafer, Dan Frankowski. 2007. Collaborative Filtering Recommender Systems. Lecture Notes in Computer Science (LNCS) 4321 (2007), 291, 324. DOI : http://dx.doi.org/10.1007/978-3-540-72079-9_9

[5] Marius Kaminskas and Derek Bridge. 2016. Diversity, Serendipity, Novelty, and Coverage: A Survey and Empirical Analysis of Beyond-Accuracy Objectives in Recommender Systems. ACM Trans. Interact. Intell. Syst. 7, 1, Article 2 (Dec. 2016), 42 pages. DOI : http://dx.doi.org/10.1145/2926720

[6] Pasquale Lops, Marco De Gemmis, and Giovanni Semeraro. 2011. Content-based recommender systems: State of the art and trends. In Recommender systems handbook. Springer, 73-105.

[7] Dunja Mladenic. July 1999. Text-Learning and Related Intelligent Agents: A Survey. IEEE Intelligent Systems 14 (July 1999), 44-54. DOI : http://dx.doi.org/10. $1109 / 5254.784084$

[8] Xia Ning and George Karypis. 2012. Sparse Linear Methods with Side Information for Top-N Recommendations. In Proceedings of the 21st International Conference on World Wide Web (WWW'12 Companion). ACM, New York, NY, USA, 581-582. DOI : http://dx.doi.org/10.1145/2187980.2188137

[9] Yannis Manolopoulos Panagiotis Symeonidis, Alexandros Nanopoulos. 2007. Feature-Weighted User Model for Recommender Systems. UM 07 Proceedings of the 11th international conference on User Modeling (2007), 97 - 106. DOI: 
http://dx doi.org/10.1007/978-3-540-73078-1_13

[10] Steffen Rendle. 2012. Factorization Machines with libFM. ACM Trans. Intell. Syst. Technol. 3, 3, Article 57 (May 2012), 22 pages. DOI: http://dx.doi.org/10.1145/
2168752.2168771

[11] George Karypis Xia Ning. 2011. SLIM: Sparse Linear Methods for Top-N Recommender Systems. IEEE. DOI : http://dx.doi.org/10.1109/ICDM.2011.134 